CORRECTION

https://doi.org/10.1038/s41586-018-0297-6

\title{
Publisher Correction: Magnetic edge states and coherent manipulation of graphene nanoribbons
}

Michael Slota, Ashok Keerthi, William K. Myers,

Evgeny Tretyakov, Martin Baumgarten, Arzhang Ardavan, Hatef Sadeghi, Colin J. Lambert, Akimitsu Narita,

Klaus Müllen \& Lapo Bogani

Correction to: Nature https://doi.org/10.1038/s41586-018-0154-7, published online 30 May 2018.

In Fig. 1 of this Letter, owing to an error during the production process, there should have been two nitrogen $(\mathrm{N})$ atoms at the 1,3-positions of all the blue chemical structures (next to the oxygen atoms), rather than one at the 2-position. The figure has been corrected online, and the original incorrect figure is shown as Supplementary Information to this Amendment, for transparency.

Supplementary information is available for this Amendment at https://doi.org/ 10.1038/s41586-018-0297-6 